\title{
Adaptive Active Control of Machine-Tool Vibration in a Lathe
}

\author{
I. Claesson ${ }^{(1)}$ and L. Håkansson ${ }^{(2)}$ \\ (I) Department of Signal Processing, University of Karlskrona/Ronneby, 37225 Ronneby, Sweden \\ (2) Department of Production and Materials Engineering, Lund University, Box 118, 22100 Lund, Sweden
}

\begin{abstract}
In turning operations the relative dynamic motion between the cutting tool and workpiece, or vibration, is a frequent problem. This affects the results of the machining, and in particular, the surface finish. Tool life is also influenced by vibration. Noise in the working environment frequently occurs as a result of dynamic motion between the cutting tool and the workpiece. With proper machine design, i.e. improved stiffness of the machine structure, the problem of relative dynamic motion between the cutting tool and workpiece may be partially solved. However, by use of active control of the machine-tool vibration, a further reduction of the dynamic motion between the cutting tool and workpiece can be achieved. It was found that adaptive feedback control of the tool vibration in the cutting speed direction, based on the fil-tered-x LMS-algorithm, enables a reduction in vibration, by up to $40 \mathrm{~dB}$ at $1.5 \mathrm{kHz}$, and by approximately $40 \mathrm{~dB}$ at $3 \mathrm{kHz}$. It was also observed that the introduction of leakage in the filtered-x LMS-algorithm improved the stability properties of the feedback control system. A significant improvement in the workpiece surface finish was observed and a substantial reduction in the noise level was obtained with adaptive control.
\end{abstract}

\section{INTRODUCTION}

In turning operations the tool and tool holder shank are subjected to dynamic excitation due to the deformation of the work material during the cutting operation. The stochastic chip formation process usually induces vibrations in the machine-tool system. Energy from the chip formation process excites the mechanical modes of the machine-tool system. Modes of the workpiece may also influence tool vibration. The relative dynamic motion between the cutting tool and workpiece will affect the result of the machining, in particular the surface finish. Furthermore, the tool life is correlated with the amount of vibration and the acoustic noise introduced. The noise is sometimes almost unbearable for the operator.

It is well known that vibration problems are closely related to the dynamic stiffness of the structure of the machinery and workpiece material. The vibration problem may be solved in part by proper machine design leading to a stiffer machine structure. In order to achieve further improvements the dynamic stiffness of the tool holder shank can be increased more selectively. One solution to these problems is active control of the tool vibrations.

Generally, machine-tool systems are classified as narrowband systems ${ }^{1}$ and as a consequence tool shank vibrations can usually be described as a superposition of narrow-band random processes at each modal frequency. These when added together form a more wide-band random process. ${ }^{1}$ The tool vibrations in a turning operation mainly comprise vibrations in two directions: the cutting speed direction and the feed direction. ${ }^{1,2}$ Usually, the vibrations in the cutting speed direction and the feed direction are linearly independent, except at some of the eigenfrequencies. ${ }^{1}$ Consequently, the control problem involves the introduction of two secondary sources, driven in such a way that the anti-vibrations generated by means of these sources interfere destructively with the tool vibration. ${ }^{3}$ However, in external longitudinal turning, most of the vibration energy is usually induced in the cutting speed direction. ${ }^{1,2}$ It is thus likely that the control of tool vibration in the cutting speed direction is an adequate solution to the vibration problem. ${ }^{2,4}$ A complication in the turning operation is that the original excitation of the tool vibration in the chip formation process cannot be observed directly and thus cannot be used as a feedforward control signal.

The statistical properties of the tool vibration imply a controller which utilizes the statistical correlation of the vibrations. ${ }^{5}$ A classical statistical criterion is the mean square error criterion. ${ }^{6}$ However, a controller based on this criterion cannot generally solve the control problem, since such a controller is only "optimum" in a stationary environment. The statistical properties of the tool vibrations may vary during the machining process. Changes in cutting data and material properties influence the statistical properties of tool vibrations. ${ }^{1,2}$ Variation within the allowed cutting data interval may also influence the structural response of the tool holder. ${ }^{2}$ In the case of constant cutting data, adaptive feedback control of machine-tool vibration based on the well known filtered-x LMS-algorithm seems very promising. ${ }^{2}$ However, variations within the excitation and the structural response of the tool holder influence the stability of the adaptive feedback control system. The stability of the feedback control system is affected by the ability of the filtered-x LMS-algorithm to control the adaptive FIR filter, the time varying controller response, without violating the closed-loop stability requirements, i.e. the Nyquist stability criterion. ${ }^{8}$ A solution to the control problem is to control the adaptive FIR filter with the leaky version of the filtered-x LMS-algorithm. ${ }^{4}$

This paper discusses single-channel feedback control of tool vibration in the cutting speed direction. The single channel control system is illustrated in Fig. 1.

The tool holder in this application has integrated actuators, i.e. secondary sources, which have been developed at DPME (Department of Production and Materials Engineer- 